\title{
Differential patterns of metastatic dissemination across medulloblastoma subgroups
}

\author{
*Michal Zapotocky, MD, PhD, ${ }^{1,2}$ Daddy Mata-Mbemba, MD, PhD, ${ }^{3}$ David Sumerauer, MD, ${ }^{2}$ \\ Petr Liby, MD, PhD, ${ }^{4}$ Alvaro Lassaletta, MD, PhD, ${ }^{1}$ Josef Zamecnik, MD, ${ }^{5}$ \\ Lenka Krskova, PhD, ${ }^{5}$ Martin Kyncl, MD, ${ }^{6}$ Jan Stary, MD, ${ }^{2}$ Suzanne Laughlin, MD, ${ }^{3}$ \\ Anthony Arnoldo, PhD, ${ }^{7}$ Cynthia Hawkins, MD, PhD, ${ }^{7}$ Uri Tabori, MD, ${ }^{1}$ Michael D. Taylor, MD, PhD, ${ }^{8}$ \\ Eric Bouffet, MD, ${ }^{1}$ Charles Raybaud, MD, ${ }^{3}$ and Vijay Ramaswamy, MD, PhD ${ }^{1,9}$
}

\begin{abstract}
1Department of Paediatrics, Division of Haematology/Oncology, ${ }^{3}$ Department of Diagnostic Imaging, Division of Neuroradiology, ${ }^{7}$ Department of Paediatric Laboratory Medicine, ${ }^{8}$ Division of Neurosurgery, and ${ }^{9}$ Programme in Neuroscience and Mental Health, University of Toronto and The Hospital for Sick Children, Toronto, Ontario, Canada; and Departments of ${ }^{2}$ Pediatric Hematology and Oncology, ${ }^{4}$ Neurosurgery, ${ }^{5}$ Pathology and Molecular Medicine, and ${ }^{6}$ Radiology, 2 nd Faculty of Medicine, Charles University and University Hospital Motol, Prague, Czech Republic
\end{abstract}

OBJECTIVE Metastatic dissemination is a major treatment challenge and cause of death in patients with medulloblastoma. However, the influence of molecular biology on the pattern of metastatic dissemination at diagnosis is not known. In this study, the authors sought to define the location, pattern, and imaging characteristics of medulloblastoma metastases across subgroups at diagnosis.

METHODS A consecutive cohort of patients with metastatic medulloblastoma at The Hospital for Sick Children and the University Hospital Motol, who underwent up-front MRI of the craniospinal axis, was assembled and allocated to subgroups using NanoString limited gene-expression profiling. Radiological characteristics (including location, morphology, size, diffusion restriction, and contrast enhancement) were discerned through a retrospective review.

RESULTS Forty metastatic medulloblastomas were identified with up-front neuroimaging of the craniospinal axis: 5 sonic hedgehog (SHH), 16 Group 3, and 19 Group 4 metastases. Significant subgroup-specific differences were observed, particularly with respect to tumor location, size, and morphology. Group 3 metastases were most frequently laminar compared with a more nodular pattern in Group 4 (14 of 16 in Group 3 vs 8 of 19 in Group 4; $p=0.0004$ ). Laminar metastases were not observed in patients with SHH medulloblastoma. Suprasellar metastases are highly specific to Group $4(p=0.016)$. Two of the $5 \mathrm{SHH}$ cases had multifocal lesions in the cerebellum, raising the possibility that these were in fact synchronous primary tumors and not true metastases. A minority of patients with Group 4 metastases harbored metastatic deposits that did not enhance on MRI after contrast administration, often in patients whose primary tumor did not enhance.

CONCLUSIONS The location, morphology, and imaging characteristics of metastatic medulloblastoma differ across molecular subgroups, with implications for diagnosis and management. This suggests that the biology of leptomeningeal dissemination differs among medulloblastoma subgroups.

https://thejns.org/doi/abs/10.3171/2017.8.PEDS17264

KEY WORDS medulloblastoma; metastasis; molecular subgroups; MRI; oncology

$\mathrm{M}$ EDULLOBLASTOMA is the most common malignant brain tumor in children. ${ }^{14,20,28,33}$ Although historically considered 1 disease, it is now abundantly clear that it comprises 4 distinct entities, with distinct demographic features, outcomes, and, in some instances, cells of origin. 1,2,21,22,26,31 These 4 distinct subgroupstermed wingless (WNT), sonic hedgehog (SHH), Group 3, and Group 4-also have distinct cells of origin; $\mathrm{SHH}$ medulloblastomas arise from the external granule layer and WNT tumors arise from the lower rhombic lip. $6,8,18,23$

ABBREVIATIONS SHH = sonic hedgehog; $\mathrm{WNT}=$ wingless.

SUBMITTED May 16, 2017. ACCEPTED August 8, 2017.

INCLUDE WHEN CITING Published online December 8, 2017; DOI: 10.3171/2017.8.PEDS17264.

* Drs. Zapotocky and Mata-Mbemba contributed equally to this work and share first authorship. Drs. Raybaud and Ramaswamy contributed equally to this work and share senior authorship. 
In the clinical setting, medulloblastoma subgroups can be reliably established using expression profiling of a limited gene panel or DNA methylation profiling. . $^{15,22,25,29,30}$

Because a full expression array or genome-wide methylation array is expensive and not clinically established, surrogate markers for molecular subgroups have been investigated. Previously, several groups reported subgroupspecific differences in MRI characteristics across the primary site of medulloblastomas. Specifically, WNT tumors arise mainly from lateral recesses (or cerebellopontine angle) in contrast to SHH tumors, the majority of which occupy cerebellar hemispheres. ${ }^{17,19,24,27,34}$ Group 3 and 4 tumors occupy the fourth ventricle, where Group 4 tumors frequently do not display contrast enhancement. ${ }^{19,27}$ This suggests that MRI is a promising tool for noninvasive determination of molecular subgroup and is probably a reflection of distinct cells of origin for each of the subgroups. ${ }^{6,23}$

Metastatic disease has occurred in $20 \%-30 \%$ of all medulloblastoma cases at diagnosis and is associated with poorer prognosis. ${ }^{4,21,32,36}$ Metastatic dissemination in medulloblastoma is largely exclusive to the leptomeninges; however, the mechanisms of dissemination are poorly understood. The incidence of metastatic dissemination is also highly subgroup specific; metastatic dissemination at diagnosis is highly enriched in Group 3 and 4 tumors and relatively rare in WNT and SHH medulloblastomas., , $10,21,31^{2}$

We have previously shown that the pattern of relapse is highly subgroup specific: SHH medulloblastomas recur more frequently in the previously treated resection cavity, whereas Group 3 and 4 tumors recur almost exclusively with metastatic dissemination. ${ }^{23}$ Moreover, isolated metastatic focal relapses are more frequently observed in Group 4 tumors, whereas diffuse multifocal or laminar relapses are more frequently seen in Group $3 .{ }^{23}$ However, there is a paucity of data on the pattern of metastatic dissemination at diagnosis. It has been shown that metastatic spread at diagnosis is heterogeneous, suggesting that this heterogeneity in the pattern of metastatic dissemination at diagnosis is probably secondary to underlying biological differences. ${ }^{3}$

Considering the significantly increased rate of treatment failure in the metastatic compartment across the majority of patients with medulloblastoma, it is crucial to understand the pattern of metastatic dissemination at diagnosis. The distinct subgroup-specific neuroimaging characteristics of the primary tumor suggest that there are differences in the pattern of metastatic dissemination. To discern the subgroup-specific patterns of metastatic dissemination in medulloblastoma, we assembled a large cohort of patients with metastatic medulloblastoma and correlated the characteristics of the metastatic disease by subgroup. We found that the pattern of metastatic dissemination at diagnosis is highly subgroup specific.

\section{Methods}

\section{Patient Cohorts}

A cohort of consecutive patients with metastatic medulloblastoma diagnosed between January 2000 and December 2016 at The Hospital for Sick Children (Toronto,
Ontario, Canada) and University Hospital Motol (Prague, Czech Republic) was retrospectively evaluated. Inclusion criteria consisted of available diagnostic MR images of the brain and spine before surgery and a sufficient amount of fresh-frozen or formalin-fixed paraffin-embedded tissue for medulloblastoma subgrouping. The study was conducted with approval from the Research Ethics Board at The Hospital for Sick Children, with a waiver of informed consent.

\section{Medulloblastoma Subgrouping}

Medulloblastoma subgrouping was performed as previously described using a 22-gene classifier. ${ }^{15}$

\section{Review of Imaging}

Diagnostic MR images obtained before surgery were independently evaluated by 2 experienced neuroradiologists who were blinded to molecular subgroup. Features of primary tumor as well as metastatic lesions were evaluated. In the primary tumor, the following features were reviewed: tumor location, tumor size (3 perpendicular diameters measured on FLAIR sequence), and contrast enhancement. Tumors were considered nonenhancing if less than one-third of the tumor volume was enhancing. Metastatic lesions were evaluated for location, enhancement, diffusion restriction, and pattern of spread (laminar vs nodular). As previously published, metastatic spread was defined as nodular only when all lesions were measurable. ${ }^{3}$ A combination of nodular and laminar metastases was recorded as laminar.

\section{Statistical Analysis}

Statistical analysis of categorical data was performed using Fisher's exact test in the R Statistical Environment (version 3.3.2).

\section{Results}

\section{Demographic Data of the Metastatic Medulloblastoma Cohorts}

Between January 2000 and December 2016, 158 cases of medulloblastoma were diagnosed at The Hospital for Sick Children. Complete diagnostic imaging (MRI of brain and spine-brain before surgery and spine before or after surgery) and molecular subgrouping were available in 117 patients. Of these 117 patients, 27 (23\%) presented with visible metastatic disease, consistent with previous reports of the incidence of metastatic dissemination in medulloblastoma (Tables 1 and 2, Toronto cohort). The incidence of metastatic dissemination varied significantly across the 4 subgroups, at distributions consistent with those of previous studies $(\mathrm{p}=0.0197)$. As expected, no metastatic WNT tumors were identified in our cohort. Three of 26 (11.5\%) patients with SHH medulloblastoma had metastatic disease, 10 of 28 (35.7\%) had Group 3, and 14 of 46 (30.4\%) had Group 4.

To validate our findings in an independent nonoverlapping cohort, we assembled a cohort of 13 patients with metastatic medulloblastomas diagnosed and treated at the University Hospital Motol (Prague cohort) between 
TABLE 1. Characteristics of primary tumors and metastases across medulloblastoma subgroups

\begin{tabular}{|c|c|c|c|c|}
\hline Variable & $\mathrm{SHH}$ & Group 3 & Group 4 & p Value* \\
\hline No. of cases $(n=40)$ & 5 & 16 & 19 & \\
\hline Primary tumor size, $\mathrm{mm}$ & & & & 0.0001 \\
\hline$<35$ & 0 & 9 & 0 & \\
\hline$\geq 35$ & 5 & 7 & 19 & \\
\hline Location & & & & 0.001 \\
\hline 4th ventricle/midline & 2 & 16 & 16 & \\
\hline Hemisphere & 3 & 0 & 0 & \\
\hline Lateral recess & 0 & 0 & 3 & \\
\hline Enhancement & & & & 0.003 \\
\hline $\mathrm{C}+$ & 5 & 15 & 9 & \\
\hline C- & 0 & 1 & 10 & \\
\hline Metastases (character) & & & & 0.0004 \\
\hline Nodular only & 5 & 2 & 11 & \\
\hline Laminar & 0 & 14 & 8 & \\
\hline Metastases (location)† & & & & 0.166 \\
\hline Supratentorial & 0 & 8 & 13 & \\
\hline Suprasellar & 0 & 0 & 9 & 0.016 \\
\hline Infratentorial & 3 & 11 & 9 & \\
\hline Spinal & 2 & 15 & 10 & \\
\hline Metastases (enhancement) & & & & 0.775 \\
\hline $\mathrm{C}+$ & 5 & 15 & 16 & \\
\hline C- & 0 & 1 & 3 & \\
\hline
\end{tabular}

$\mathrm{C}+=$ contrast enhancement; $\mathrm{C}-=$ no contrast enhancement.

* The $p$ values were determined using Fisher's exact test.

$\dagger$ The sum is $>40$ because each case could have metastatic disease in 1-3 compartments.

January 2000 and December 2015. The Prague cohort comprised 2 SHH cases, 6 Group 3 cases, and 5 Group 4 cases, similar in distribution to the Toronto cohort $(\mathrm{p}=$ 0.72). Because the imaging characteristics and distribution of metastatic dissemination by subgroup were similar, we combined the cohorts in our analysis (Tables 2 and 3).

\section{MRI Characteristics of the Primary Tumor in Metastatic Medulloblastoma Differ by Subgroup}

The appearance of primary site medulloblastoma in the setting of metastatic dissemination differed by subgroup (Table 1). The location in the cerebellar hemispheres was typical for SHH medulloblastoma and was not observed in other subgroups. In contrast, 3 cases of Group 4 medulloblastoma exhibited locations in a lateral recess that was initially reported as being pathognomonic for the WNT subgroup..$^{17}$ The remaining 16 cases of Group 4 and all Group 3 tumors were located in the fourth ventricle (midline) $(\mathrm{p}=0.001)$. Strikingly, we observed a significant difference in the size of primary tumors between metastatic Group 3 and 4 tumors. This specific feature was present uniquely in Group 3, where in 56\% of cases the largest diameter of the tumor did not exceed $35 \mathrm{~mm}$ compared with other subgroups ( $\mathrm{SHH}$ and Group 4 tumors were all $>35$ $\mathrm{mm}$ ) (Fig. 1; $\mathrm{p}=0.0001)$. Consistent with previous obser-
TABLE 2. Characteristics of primary tumors and metastases across medulloblastoma subgroups (Toronto cohort only)

\begin{tabular}{|c|c|c|c|c|}
\hline Variable & $\mathrm{SHH}$ & Group 3 & Group 4 & $p$ Value* \\
\hline No. of cases $(n=27)$ & 3 & 10 & 14 & \\
\hline Size of primary tumor, $\mathrm{mm}$ & & & & 0.00013 \\
\hline$<35$ & 0 & 7 & 0 & \\
\hline$\geq 35$ & 3 & 3 & 14 & \\
\hline Tumor location & & & & 0.009 \\
\hline 4th ventricle/midline & 1 & 10 & 12 & \\
\hline Hemisphere & 2 & 0 & 0 & \\
\hline Lateral recess & 0 & 0 & 2 & \\
\hline Enhancement & & & & 0.119 \\
\hline $\mathrm{C}+$ & 3 & 9 & 8 & \\
\hline C- & 0 & 1 & 6 & \\
\hline Metastases (character) & & & & 0.0408 \\
\hline Nodular only & 3 & 2 & 8 & \\
\hline Laminar & 0 & 8 & 6 & \\
\hline Metastases (location)† & & & & 0.532 \\
\hline Supratentorial & 0 & 5 & 10 & \\
\hline Suprasellar & 0 & 0 & 7 & 0.138 \\
\hline Infratentorial & 2 & 7 & 7 & \\
\hline Spinal & 1 & 9 & 8 & \\
\hline Metastases (enhancement) & & & & 1.0 \\
\hline $\mathrm{C}+$ & 3 & 9 & 12 & \\
\hline C- & 0 & 1 & 2 & \\
\hline
\end{tabular}

* The $p$ values were determined using Fisher's exact test.

† The sum is $>27$ because each case could have metastatic disease in 1-3 compartments.

vations, the pattern of enhancement was also different in Group 4 than in other subgroups, in particular the proportion of nonenhancing primary tumors $(52.6 \% ; \mathrm{p}=0.003)$.

\section{Anatomical Location of Metastatic Dissemination Is Highly Subgroup Specific}

We next investigated if the anatomical pattern of metastatic deposits has subgroup specificity. As such, we divided the location of metastases among the supratentorial, infratentorial, and spinal compartments, as well as by laminar versus nodular, as previously reported. ${ }^{3}$ Surprisingly, no significant difference in distribution of metastatic lesions per compartment was identified (Table 1). Interestingly, only 3 of 16 Group 3 cases (18.7\%), 3 of 19 Group 4 (15.8\%), and 2 of 5 SHH (40\%) presented with purely spinal metastases.

When we analyzed the anatomical location of metastasis by subgroup, we found that there were considerable differences among the 3 subgroups. The majority of spinal metastases occurred as part of a diffuse leptomeningeal spread and did not have a subgroup specificity ( 27 of 40 cases). However, 9 of 19 patients (47.4\%) with Group 4 medulloblastoma exhibited the presence of metastasis in the anterior part of the third ventricle (infundibulum/recess) (Fig. 2A and B). Suprasellar metastasis was either a solitary lesion (5 of 9 cases) or was part of more extensive 
TABLE 3. Characteristics of primary tumors and metastases across medulloblastoma subgroups (Prague cohort only)

\begin{tabular}{|c|c|c|c|c|}
\hline Variable & $\mathrm{SHH}$ & Group 3 & Group 4 & $p$ Value* \\
\hline No. of cases $(n=13)$ & 2 & 6 & 5 & \\
\hline Size of primary tumor, $\mathrm{mm}$ & & & & 0.62 \\
\hline$<35$ & 0 & 2 & 0 & \\
\hline$\geq 35$ & 2 & 4 & 5 & \\
\hline Tumor location & & & & 0.14 \\
\hline 4th ventricle/midline & 1 & 6 & 4 & \\
\hline Hemisphere & 1 & 0 & 0 & \\
\hline Lateral recess & 0 & 0 & 1 & \\
\hline Enhancement & & & & 0.007 \\
\hline $\mathrm{C}+$ & 2 & 6 & 1 & \\
\hline C- & 0 & 0 & 4 & \\
\hline Metastases (character) & & & & 0.02 \\
\hline Nodular only & 2 & 0 & 3 & \\
\hline Laminar & 0 & 6 & 2 & \\
\hline Metastases (location)† & & & & 0.526 \\
\hline Supratentorial & 0 & 3 & 3 & \\
\hline Suprasellar & 0 & 0 & 2 & 0.67 \\
\hline Infratentorial & 1 & 4 & 2 & \\
\hline Spinal & 1 & 6 & 2 & \\
\hline Metastases (enhancement) & & & & 0.99 \\
\hline $\mathrm{C}+$ & 2 & 6 & 4 & \\
\hline C- & 0 & 0 & 1 & \\
\hline
\end{tabular}

* The $p$ values were determined using Fisher's exact test.

$\dagger$ The sum is $>13$ because each case could have metastatic disease in 1-3 compartments.

metastatic disease (4 of 9 cases). Suprasellar metastasis clearly delineated Group 4 medulloblastoma because it was not observed in any of the other molecular subgroups $(\mathrm{p}=0.016)$.

Among other subgroups, only 2 patients with Group 3 medulloblastoma (12.5\%) presented with large metastatic lesions that occupied the whole third ventricle and that were not limited to the suprasellar region. Interestingly, both of these patients presented with primary disease that was smaller $(<35 \mathrm{~mm})$ than the metastatic lesion (Figs. 1, 2B, and 2C). Two patients with SHH medulloblastoma presented with metastatic nodular disease in the cerebellum, seemingly outside the primary tumor (Fig. 3) and without other more typical leptomeningeal metastases, raising suspicion that these may have in fact represented synchronous primary tumors rather than a single tumor that had disseminated.

\section{Subset of Metastatic Deposits Does Not Enhance but Restricts Diffusion}

Primary Group 4 tumors have been shown to have less gadolinium uptake. ${ }^{19,27}$ This raises the question of whether the metastatic deposits in Group 4 tumors also do not enhance with gadolinium. To determine if there is a discrepancy between the enhancement patterns of the metastatic and primary compartments, we analyzed contrast uptake

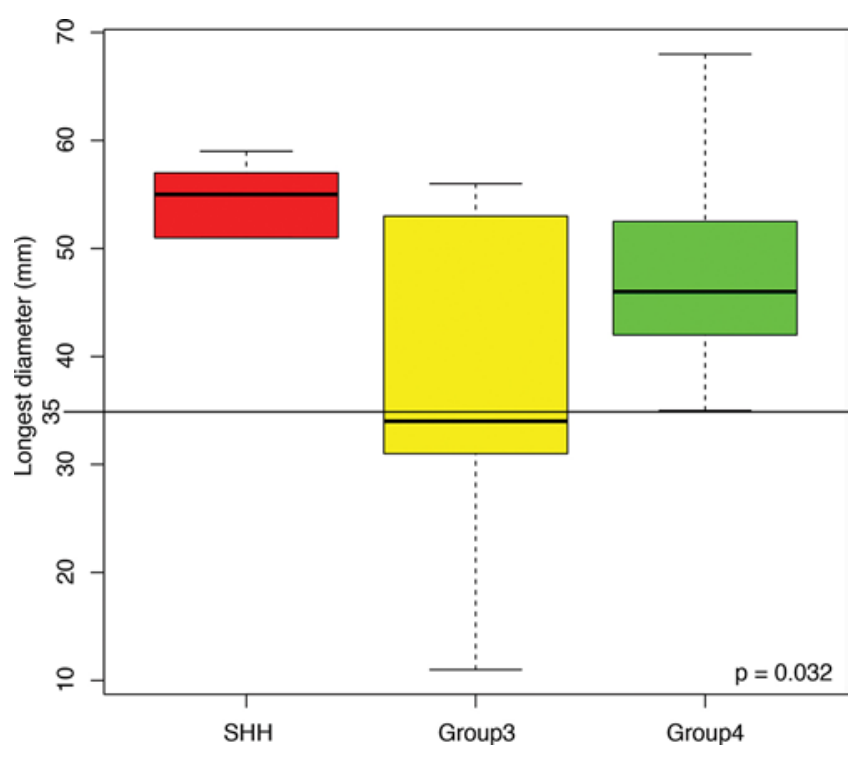

FIG. 1. Box plots depicting differences in primary tumor size among molecular subgroups. The solid line represents the longest diameter of 35 $\mathrm{mm}$. The $p$ value was calculated using a nonparametric Kruskal-Wallis test. Figure is available in color online only.

of the metastases and compared it to that of the primary tumor. Although the majority of tumors, including Group 4 , were contrast enhancing, we found that 4 of $40(10 \%)$ had reduced or absent contrast enhancement. One case was a Group 3 medulloblastoma with a nonenhancing primary tumor and nonenhancing metastases in the spinal canal. The other 3 patients with nonenhancing Group 4 primary tumors presented with nonenhancing metastases in the suprasellar region and cerebellum, respectively (Fig. $4 \mathrm{~A}$ and $\mathrm{B})$.

An additional 4 patients with Group 4 tumors exhibited an unexpected pattern of enhancement, with some metastases enhancing and some nonenhancing. Strikingly, 1 metastatic lesion could be partially enhancing, with the rest of the lesion remaining nonenhanced. Importantly, all intracranial metastatic lesions, either enhancing or nonenhancing, exhibited diffusion restriction on diffusionweighted imaging and apparent diffusion coefficient (Fig. 4C and D). This strongly suggests that diffusion-weighted imaging is crucial to fully evaluate the distribution, incidence, and frequency of metastatic dissemination in Group 3 and 4 tumors.

\section{Laminar Metastases Are More Common in Group 3 Tumors}

All patients with SHH medulloblastomas had nodular metastases, in contrast to cases involving Group 3 tumors, where $87.5 \%$ of cases (14 of 16) exhibited laminar metastases. Eleven of 19 patients with Group 4 tumors had nodular metastases. ${ }^{3}$ Altogether, the morphology of metastases differed significantly across subgroups $(\mathrm{p}=0.00002)$.

\section{Discussion}

Herein we report, to our knowledge, the largest cohort of patients with metastatic medulloblastoma profiled at 

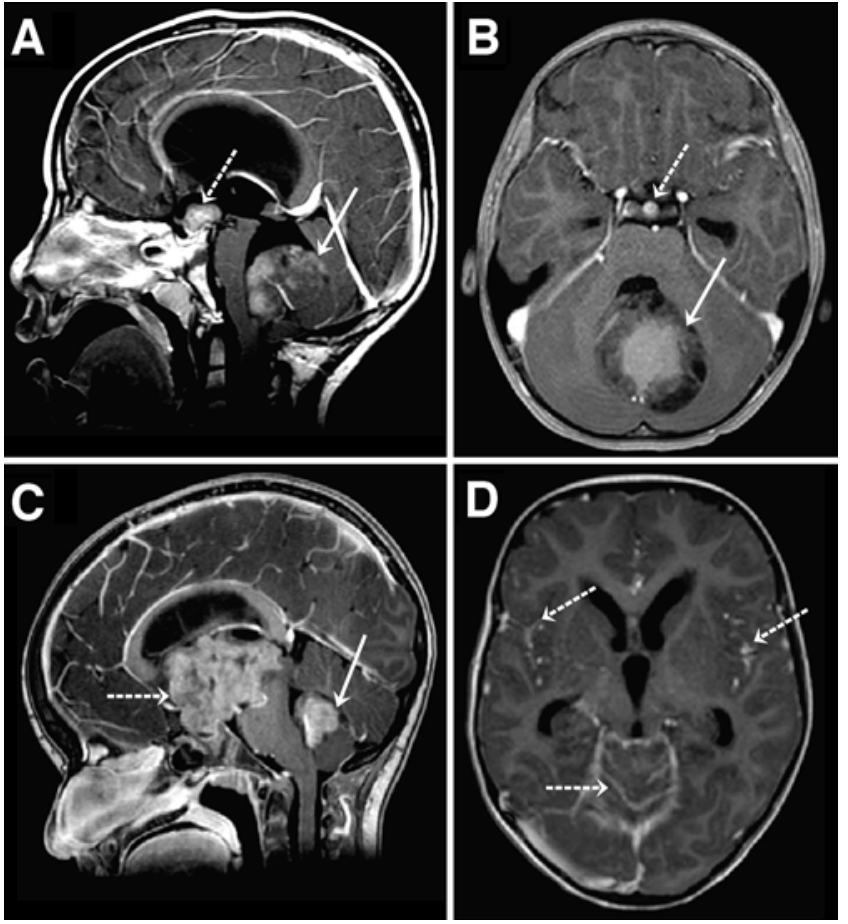

FIG. 2. MRI characteristics of Group 3 ( $A$ and $B$ ) and Group 4 ( $C$ and D) medulloblastoma. A: Postgadolinium sagittal T1-weighted image demonstrating a poorly enhancing primary tumor in the posterior fossa and a nodular enhancing lesion in the suprasellar region (with less than one-third of the tumor enhancing and therefore defined as nonenhancing). B: Postgadolinium axial T1-weighted image demonstrating a heterogeneously enhancing primary tumor in the posterior fossa and a small nodular enhancing lesion in the suprasellar region. C: Postgadolinium sagittal T1-weighted image showing a small fourth ventricular primary tumor and a large metastasis filling the third ventricle. D: Postgadolinium axial T1-weighted image showing diffuse leptomeningeal dissemination in the infratentorial and supratentorial compartments. In all images, solid arrows show primary tumor and dashed arrows show metastatic lesions.

diagnosis established by combined MRI and molecular subgrouping. We found important subgroup-specific differences in the pattern of metastatic dissemination, which
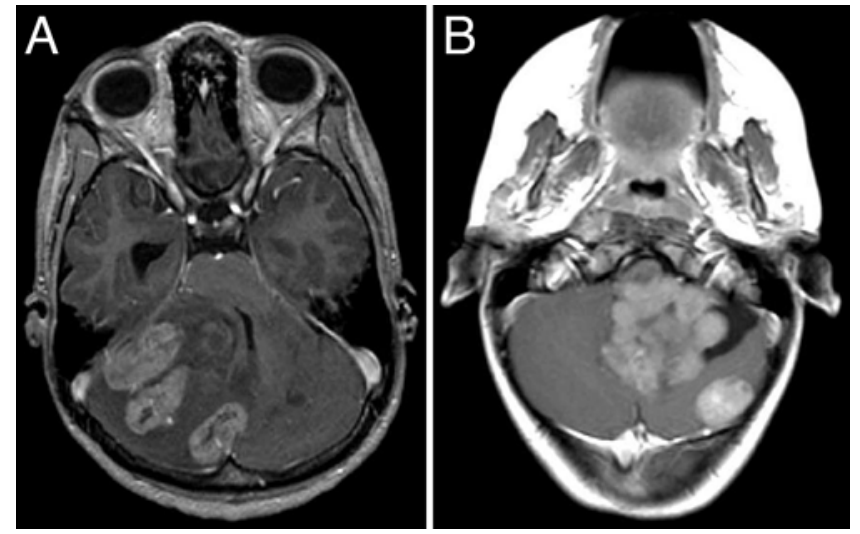

FIG. 3. Multifocal appearance of posterior fossa metastatic involvement in SHH medulloblastoma cases. A and B: Postgadolinium axial T1-weighted images obtained in 2 patients with $\mathrm{SHH}$ medulloblastoma presenting with posterior fossa metastases.
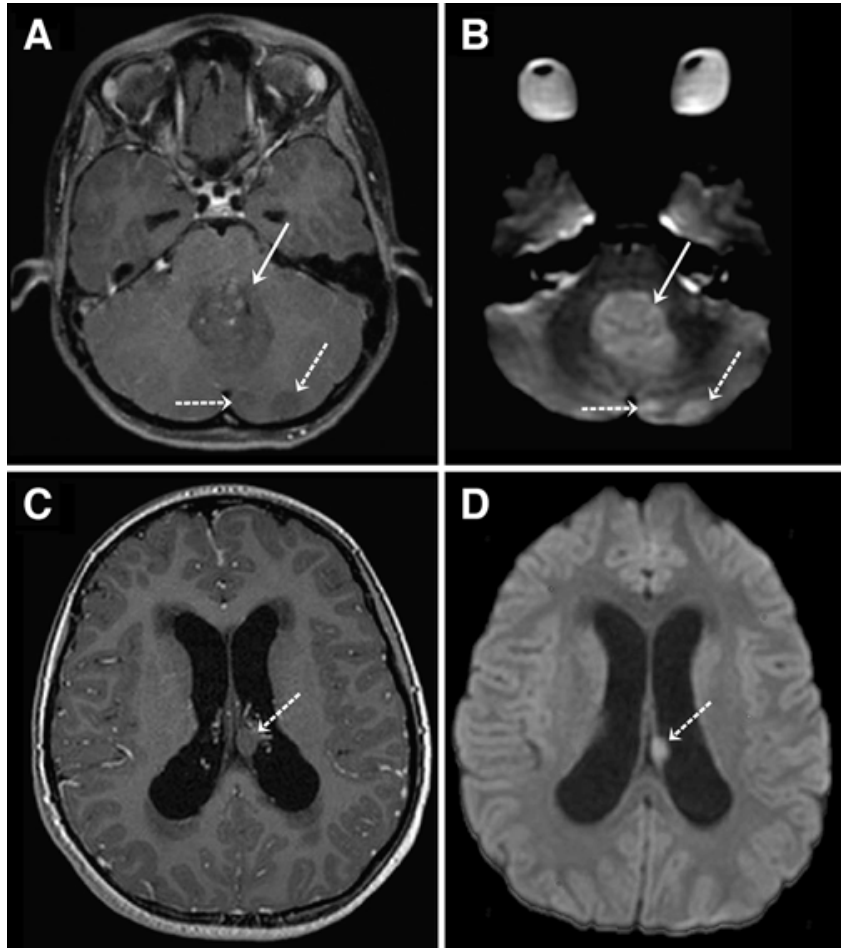

FIG. 4. Diffusion restriction on diffusion-weighted imaging in nonenhancing metastatic lesions. Images were obtained in a patient with Group 4 medulloblastoma and posterior fossa metastases (A and B) and a patient with Group 4 medulloblastoma with supratentorial metastases (C and D). A: Postgadolinium axial T1-weighted image demonstrating a nonenhancing primary tumor centered within the fourth ventricle and nonenhancing metastases in the left cerebellum posteriorly. B: Axial B1000 image demonstrating diffusion restriction in all visible lesions. C: Postgadolinium axial T1-weighted image demonstrating nonenhancing metastasis in the left lateral ventricle. D: Axial B1000 image demonstrating diffusion restriction in a left lateral ventricle metastasis. In all images, solid arrows show primary tumor and dashed arrows show metastatic lesions.

have a significant impact on future clinical and preclinical studies of medulloblastoma. First, we observed that Group 3 primary tumors are frequently smaller in the presence of metastases. Second, we found that the morphology and anatomical location are highly subgroup specific, with Group 4 tumors having a specific proclivity for the suprasellar space. Finally, we found that a small but significant proportion of metastatic deposits do not exhibit gadolinium uptake, suggesting that diffusion restriction is crucial to diagnosing or excluding leptomeningeal disease.

Interestingly, 2 of 5 patients with metastatic SHH medulloblastoma exhibited intraparenchymal nodular lesions in the cerebellum adjacent to the primary tumor in the posterior fossa. This raises the possibility that these are synchronous primary tumors as opposed to true metastases, supported by the fact that intraparenchymal metastases are almost nonexistent in medulloblastoma. With advances in next-generation sequencing, it will be crucial to sample these multifocal lesions in the cerebellum and to determine if these are indeed clonal or if they display branched evolution, as would be expected with a true metastasis, and also if they occur in the setting of a germline 
mutation of PTCH or SUFU. The issue of synchronous tumors compared with true metastatic disease in these patients has profound implications, because they currently receive 36-39 Gy of craniospinal radiation for putative metastatic disease; as such, these patients have a higher risk of significant long-term neurocognitive morbidity as the result of treatment. ${ }^{12,13}$

It is of tremendous clinical importance that in a small minority of cases, metastatic Group 4 disease did not show enhancement after gadolinium administration. This has major implications for the design of clinical trials, whereas a reliance on enhancing metastatic disease will result in a significant underdiagnosis of high-risk medulloblastoma. Previous studies have shown that administering reduced-dose craniospinal radiation to metastatic medulloblastoma results in a poor prognosis; careful examination by neuroimaging of prospective trial cohorts is required to determine if a discernable proportion of patients with nonenhancing metastatic disease is being included in average-risk strata. ${ }^{16}$

Half of Group 4 cases demonstrated metastasis in the suprasellar region at the time of diagnosis, suggesting that suprasellar metastases may be a specific marker for Group 4 medulloblastoma. A previous report demonstrated 6 cases of suprasellar metastases, albeit absent molecular subgrouping.? Herein we provide strong evidence that this location is affected by metastatic disease solely in patients with Group 4 tumors. The exact biological underpinnings of this phenomenon are unclear, but a possibility is differences in the microenvironment; specifically, the suprasellar space represents a metastatic niche more amenable to Group 4 disease. Future preclinical modeling will need to account for these differences in the microenvironment, particularly because Group 4 medulloblastoma rarely recurs in the previously radiated tumor bed. It is important to note that none of 9 patients with suprasellar metastases suffered from endocrinopathy, diabetes insipidus, or visual deterioration at the time of diagnosis, probably due to the noninfiltrative nature of Group 4 metastases.

Group 3 metastases are frequently associated with avidly enhancing small primary tumors. This was striking in our series, where in some instances the metastatic lesion was larger than the primary tumor. One possible explanation for this finding is that metastatic dissemination in Group 3 cases is an early clonal event; this is consistent with the poor prognosis for patients with metastatic Group 3 tumors who are now considered to be at high risk. ${ }^{22,35}$

This is also largely independent of MYC amplification, because only a single Group 3 medulloblastoma in the Toronto cohort harbored an amplification. This finding, coupled with the observation that Group 3 tumors fail almost exclusively with metastatic dissemination and have a tumor bed devoid of disease, is consistent with the resistance to therapy of early metastatic clones. ${ }^{23}$ Recent advances suggesting that recurrent metastatic Group 3 tumors have drastically different genomes are also consistent with this observation. ${ }^{11}$ Moving forward, routine biopsy of Group 3 metastases will help determine the true nature of clonal divergence between the primary and metastatic compartments.

Despite the small primary tumor in some cases, patients with Group 3 medulloblastoma frequently present with symptoms of hydrocephalus and, in general, a short prediagnostic interval, suggesting that their symptoms are related to metastatic disease causing distal CSF obstruction at the arachnoid granulations, rather than obstruction of CSF in the fourth ventricle. ${ }^{24}$ The significance of laminar metastases being enriched in Group 3 tumors compared with nodular metastases in Group 4 is unclear; however, the distinction is consistent with the observation that at relapse, there is a clear trend toward isolated nodular relapses being more common in Group 4 tumors. ${ }^{23}$

In addition, it is possible that the smaller primary tumors observed in Group 3 disease represent an early metastatic event leading to a more diffuse presentation. The emerging availability of robust preclinical models of Group 3 and 4 medulloblastoma may help model the events leading to this difference in the pattern of dissemination. Biologists will need to start exploring the underlying mechanisms between these different metastatic phenomena in patients with medulloblastoma. Two limitations of our study are a lack of uniform imaging protocols across all patients and blinded interobserver reliability ratings, which could be addressed in the future through analysis of prospective trial cohorts.

Clinical and research approaches to medulloblastoma metastases have largely considered them to be clinically and biologically homogeneous across subgroups. The data in the current study belie this assumption and suggest that the biology of the metastatic cascade varies among the subgroups of medulloblastoma. Overall, this study provides the first indications that the pattern of metastatic dissemination in medulloblastoma is highly subgroup specific. This study has significant clinical implications-we propose that SHH medulloblastoma presenting with possible synchronous primary tumors be sequenced to determine if branched evolution has indeed occurred and to exclude or include the presence of metastatic disease.

Furthermore, neurooncologists and neuroradiologists should be cognizant of a small proportion of nonenhancing Group 4 metastases. Overall, our findings further support the notion that the 4 subgroups are completely different diseases, where in this case they suggest that there is a distinct preference for specific microenvironments along the leptomeninges. Although this requires further functional validation in preclinical models of medulloblastoma subgroups, this suggests that therapy to the metastatic compartment will probably require a tailored subgroupspecific approach.

In the future, routine biopsy of the metastatic compartment may help to fully discern these biological differences. The current study outlines the location, morphology, and imaging characteristics of medulloblastoma metastases at diagnosis and does not describe these variables at the time of recurrence, when they deserve detailed study. Finally, our findings of subgroup-specific patterns of metastases add to the already well-described subgroup-specific radiological findings and can further refine the radiological description of the 4 subgroups, allowing for a robust presurgical diagnosis of medulloblastoma subgroup.

\section{Conclusions}

The location, morphology, and imaging characteristics of metastatic medulloblastoma differ across molecular 
subgroups, with implications for diagnosis and management. This suggests that the biology of leptomeningeal dissemination differs among medulloblastoma subgroups.

\section{Acknowledgments}

Dr. Ramaswamy is supported by grants from the American Brain Tumor Association, the Garron Family Cancer Center, Meagan's Walk, the Brain Tumour Foundation of Canada, and the Collaborative Ependymoma Research Network. Dr. Zapotocky is supported by a Garron Family Cancer Center Fellowship, the Meagan Bebenek Fellowship in Pediatric Neuro-Oncology, and Restracomp from the Research Training Center at The Hospital for Sick Children. Dr. Lassaletta is supported by the Meagan Bebenek Fellowship in Pediatric Neuro-Oncology and Restracomp from the Research Training Center at The Hospital for Sick Children. Drs. Zapotocky, Sumerauer, and Stary are supported by the project (Ministry of Health, Czech Republic) for conceptual development of research organization 00064203 (University Hospital Motol, Prague, Czech Republic). Dr. Taylor is supported by funds from the Garron Family Chair in Childhood Cancer Research at The Hospital for Sick Children and The University of Toronto and operating funds from the National Institutes of Health (R01CA159859 and R01CA148699), The Canadian Institutes of Health Research, and the Pediatric Brain Tumor Foundation. Dr. Bouffet is supported by funds from the Garron Family Chair in Childhood Cancer Research at The Hospital for Sick Children and The University of Toronto.

\section{References}

1. Cavalli FMG, Remke M, Rampasek L, Peacock J, Shih DJH, Luu B, et al: Intertumoral heterogeneity within medulloblastoma subgroups. Cancer Cell 31:737-754, 754.e1-754.e6, 2017

2. Dubuc AM, Morrissy AS, Kloosterhof NK, Northcott PA, Yu EP, Shih D, et al: Subgroup-specific alternative splicing in medulloblastoma. Acta Neuropathol 123:485-499, 2012

3. Dufour C, Beaugrand A, Pizer B, Micheli J, Aubelle MS, Fourcade A, et al: Metastatic medulloblastoma in childhood: Chang's classification revisited. Int J Surg Oncol 2012:245385, 2012

4. Gajjar A, Chintagumpala M, Ashley D, Kellie S, Kun LE, Merchant TE, et al: Risk-adapted craniospinal radiotherapy followed by high-dose chemotherapy and stem-cell rescue in children with newly diagnosed medulloblastoma (St Jude Medulloblastoma-96): long-term results from a prospective, multicentre trial. Lancet Oncol 7:813-820, 2006

5. Gerlinger M, Rowan AJ, Horswell S, Math M, Larkin J, Endesfelder D, et al: Intratumor heterogeneity and branched evolution revealed by multiregion sequencing. N Engl J Med 366:883-892, 2012

6. Gibson P, Tong Y, Robinson G, Thompson MC, Currle DS, Eden C, et al: Subtypes of medulloblastoma have distinct developmental origins. Nature 468:1095-1099, 2010

7. Helton KJ, Maron G, Mamcarz E, Leventaki V, Patay Z, Sadighi Z: Unusual magnetic resonance imaging presentation of post-BMT cerebral toxoplasmosis masquerading as meningoencephalitis and ventriculitis. Bone Marrow Transplant 51:1533-1536, 2016

8. Kawauchi D, Robinson G, Uziel T, Gibson P, Rehg J, Gao C, et al: A mouse model of the most aggressive subgroup of human medulloblastoma. Cancer Cell 21:168-180, 2012

9. Kool M, Korshunov A, Remke M, Jones DT, Schlanstein M, Northcott PA, et al: Molecular subgroups of medulloblastoma: an international meta-analysis of transcriptome, genetic aberrations, and clinical data of WNT, SHH, Group 3, and Group 4 medulloblastomas. Acta Neuropathol 123:473484, 2012
10. Lafay-Cousin L, Smith A, Chi SN, Wells E, Madden J, Margol A, et al: Clinical, pathological, and molecular characterization of infant medulloblastomas treated with sequential high-dose chemotherapy. Pediatr Blood Cancer 63:15271534,2016

11. Morrissy AS, Garzia L, Shih DJ, Zuyderduyn S, Huang X, Skowron P, et al: Divergent clonal selection dominates medulloblastoma at recurrence. Nature 529:351-357, 2016

12. Moxon-Emre I, Bouffet E, Taylor MD, Laperriere N, Scantlebury N, Law N, et al: Impact of craniospinal dose, boost volume, and neurologic complications on intellectual outcome in patients with medulloblastoma. J Clin Oncol 32:1760-1768, 2014

13. Mulhern RK, Palmer SL, Merchant TE, Wallace D, Kocak $\mathrm{M}$, Brouwers $\mathrm{P}$, et al: Neurocognitive consequences of riskadapted therapy for childhood medulloblastoma. J Clin Oncol 23:5511-5519, 2005

14. Northcott PA, Korshunov A, Pfister SM, Taylor MD: The clinical implications of medulloblastoma subgroups. Nat Rev Neurol 8:340-351, 2012

15. Northcott PA, Shih DJ, Remke M, Cho YJ, Kool M, Hawkins $\mathrm{C}$, et al: Rapid, reliable, and reproducible molecular subgrouping of clinical medulloblastoma samples. Acta Neuropathol 123:615-626, 2012

16. Packer RJ, Gajjar A, Vezina G, Rorke-Adams L, Burger PC, Robertson PL, et al: Phase III study of craniospinal radiation therapy followed by adjuvant chemotherapy for newly diagnosed average-risk medulloblastoma. J Clin Oncol 24:42024208, 2006

17. Patay Z, DeSain LA, Hwang SN, Coan A, Li Y, Ellison DW: MR Imaging characteristics of wingless-type-subgroup pediatric medulloblastoma. AJNR Am J Neuroradiol 36:23862393, 2015

18. Pei Y, Moore CE, Wang J, Tewari AK, Eroshkin A, Cho YJ, et al: An animal model of MYC-driven medulloblastoma. Cancer Cell 21:155-167, 2012

19. Perreault S, Ramaswamy V, Achrol AS, Chao K, Liu TT, Shih D, et al: MRI surrogates for molecular subgroups of medulloblastoma. AJNR Am J Neuroradiol 35:1263-1269, 2014

20. Ramaswamy V, Northcott PA, Taylor MD: FISH and chips: the recipe for improved prognostication and outcomes for children with medulloblastoma. Cancer Genet 204:577-588, 2011

21. Ramaswamy V, Remke M, Adamski J, Bartels U, Tabori U, Wang X, et al: Medulloblastoma subgroup-specific outcomes in irradiated children: who are the true high-risk patients? Neuro Oncol 18:291-297, 2016

22. Ramaswamy V, Remke M, Bouffet E, Bailey S, Clifford SC, Doz F, et al: Risk stratification of childhood medulloblastoma in the molecular era: the current consensus. Acta Neuropathol 131:821-831, 2016

23. Ramaswamy V, Remke M, Bouffet E, Faria CC, Perreault S, Cho YJ, et al: Recurrence patterns across medulloblastoma subgroups: an integrated clinical and molecular analysis. Lancet Oncol 14:1200-1207, 2013

24. Ramaswamy V, Remke M, Shih D, Wang X, Northcott PA, Faria CC, et al: Duration of the pre-diagnostic interval in medulloblastoma is subgroup dependent. Pediatr Blood Cancer 61:1190-1194, 2014

25. Ramaswamy V, Samuel N, Remke M: Can miRNA-based real-time PCR be used to classify medulloblastomas? CNS Oncol 3:173-175, 2014

26. Ramaswamy V, Taylor MD: Medulloblastoma: from myth to molecular. J Clin Oncol 35:2355-2363, 2017

27. Raybaud C, Ramaswamy V, Taylor MD, Laughlin S: Posterior fossa tumors in children: developmental anatomy and diagnostic imaging. Childs Nerv Syst 31:1661-1676, 2015

28. Remke M, Ramaswamy V, Taylor MD: Medulloblastoma 
molecular dissection: the way toward targeted therapy. Curr Opin Oncol 25:674-681, 2013

29. Schwalbe EC, Lindsey JC, Straughton D, Hogg TL, Cole M, Megahed H, et al: Rapid diagnosis of medulloblastoma molecular subgroups. Clin Cancer Res 17:1883-1894, 2011

30. Schwalbe EC, Williamson D, Lindsey JC, Hamilton D, Ryan SL, Megahed H, et al: DNA methylation profiling of medulloblastoma allows robust subclassification and improved outcome prediction using formalin-fixed biopsies. Acta Neuropathol 125:359-371, 2013

31. Taylor MD, Northcott PA, Korshunov A, Remke M, Cho YJ, Clifford SC, et al: Molecular subgroups of medulloblastoma: the current consensus. Acta Neuropathol 123:465-472, 2012

32. Thompson EM, Hielscher T, Bouffet E, Remke M, Luu B, Gururangan S, et al: Prognostic value of medulloblastoma extent of resection after accounting for molecular subgroup: a retrospective integrated clinical and molecular analysis. Lancet Oncol 17:484-495, 2016

33. Wang X, Ramaswamy V, Remke M, Mack SC, Dubuc AM, Northcott PA, et al: Intertumoral and intratumoral heterogeneity as a barrier for effective treatment of medulloblastoma. Neurosurgery 60 (Suppl 1):57-63, 2013

34. Wefers AK, Warmuth-Metz M, Pöschl J, von Bueren AO, Monoranu CM, Seelos K, et al: Subgroup-specific localization of human medulloblastoma based on pre-operative MRI. Acta Neuropathol 127:931-933, 2014

35. Wu X, Northcott PA, Dubuc A, Dupuy AJ, Shih DJ, Witt H, et al: Clonal selection drives genetic divergence of metastatic medulloblastoma. Nature 482:529-533, 2012

36. Zeltzer PM, Boyett JM, Finlay JL, Albright AL, Rorke LB, Milstein JM, et al: Metastasis stage, adjuvant treatment, and residual tumor are prognostic factors for medulloblastoma in children: conclusions from the Children's Cancer Group 921 randomized phase III study. J Clin Oncol 17:832-845, 1999

\section{Disclosures}

The authors report no conflict of interest concerning the materials or methods used in this study or the findings specified in this paper.

\section{Author Contributions}

Conception and design: Ramaswamy, Zapotocky, Taylor, Bouffet. Acquisition of data: Zapotocky, Mata-Mbemba, Sumerauer, Liby, Lassaletta, Zamecnik, Krskova, Kyncl, Stary, Laughlin, Arnoldo, Hawkins, Tabori, Raybaud. Analysis and interpretation of data: Zapotocky, Mata-Mbemba, Sumerauer, Liby, Lassaletta, Zamecnik, Krskova, Kyncl, Stary, Laughlin, Arnoldo, Hawkins, Tabori, Raybaud. Drafting the article: Zapotocky, Mata-Mbemba. Critically revising the article: Ramaswamy, Zapotocky, Laughlin, Taylor, Bouffet, Raybaud. Reviewed submitted version of manuscript: Ramaswamy, Zapotocky, Mata-Mbemba, Sumerauer, Taylor, Bouffet. Approved the final version of the manuscript on behalf of all authors: Ramaswamy. Statistical analysis: Zapotocky. Administrative/technical/material support: Sumerauer, Arnoldo. Study supervision: Ramaswamy, Raybaud.

\section{Correspondence}

Vijay Ramaswamy, Division of Haematology/Oncology, Labatt Brain Tumor Research Centre, The Hospital for Sick Children, 555 University Ave., Toronto, ON M5G 1X8, Canada. email: vijay. ramaswamy@sickkids.ca. 